\title{
Two Case Reports of Rare BRAF Mutations in Exon 11 and Exon 15 with Discussion of Potential Treatment Options
}

\author{
Georg Richtig ${ }^{a, b} \quad$ Ariane Aigelsreiter $^{c}$ Karl Kashofer $^{c} \quad$ Emina Talakic $^{d}$ \\ Romana Kupsa $^{a}$ Helmut Schaider ${ }^{\mathrm{e}}$ Erika Richtig $^{\mathrm{a}}$ \\ ${ }^{a}$ Department of Dermatology, Medical University of Graz, Graz, Austria; ${ }^{b}$ Institute of \\ Experimental and Clinical Pharmacology, Medical University of Graz, Graz, Austria; \\ 'Institute of Pathology, Medical University of Graz, Graz, Austria; ${ }^{d}$ Division of General \\ Radiological Diagnostics, Department of Radiology, Medical University of Graz, \\ Graz, Austria; ${ }^{\mathrm{e}}$ Dermatology Research Centre, School of Medicine, The University of \\ Queensland, Brisbane, QId., Australia
}

\section{Keywords}

BRAF mutation $\cdot \mathrm{K} 601 \mathrm{E} \cdot \mathrm{G} 466 \mathrm{E} \cdot \mathrm{MEK}$ inhibitor $\cdot$ Ipilimumab

\begin{abstract} mutations lead to activity levels below those of the wild type.

BRAF mutations occur in up to $50 \%$ of melanomas. Mutations in the BRAF gene directly influence the patient's treatment because several inhibitors are available that only target BRAF $^{\mathrm{V} 600}$ mutations. Herein, we describe two cases of patients with metastatic melanomas, each carrying a 'nonstandard' mutation in the BRAF gene: BRAF $^{\mathrm{K} 601 \mathrm{E}}$ and $\mathrm{BRAF}^{\mathrm{G} 466 \mathrm{E}}$, respectively. The first patient was treated with a MEK inhibitor and the second one with ipilimumab. However, not all BRAF mutations result in increased BRAF kinase activity, and clinical data for 'nonstandard' mutations, such as those described in our case report, are sparse. Therefore, treatment with MEK inhibitors can be helpful in cases where BRAF mutations result in increased activity, whereas immune checkpoint inhibitors might be used in cases where the 


\section{Case Reports in Oncology}

\section{Background}

The breakthrough discovery that mutations existed in the B-Raf proto-oncogene, serine/threonine kinase (BRAF) gene was an important step towards designing personalized treatment for patients with advanced melanoma. Between 85 and $95 \%$ of all mutations in the BRAF gene occur at codon 600 , and the most frequent mutation is a substitution of valine to glutamic acid known as BRAFV600E (COSMIC - Catalogue of somatic mutations in cancer. http://cancer.sanger.ac.uk/cosmic. Last accessed March 14, 2016) [1, 2].

Some rare BRAF mutations, apart from $\mathrm{BRAF}^{\mathrm{V} 600}$ mutations, have been detected using next-generation sequencing (NGS). This raised the question of how we should treat these patients since most of the BRAF inhibitors have only been tested in large studies in melanoma patients with BRAFV600 mutations [3-5]. As a consequence, BRAF inhibitors (e.g., vemurafenib or dabrafenib) have only been approved by the FDA for BRAFV600E melanomas and by the EMA, for all BRAF ${ }^{\mathrm{V} 600}$-mutated melanomas.

In this paper, we present two cases of patients with melanomas, each of whom had a rare BRAF mutation that was detected using NGS, which resulted in the selection of completely different therapeutic approaches.

\section{Case Presentation}

\section{Case 1}

A 69-year-old man presented himself to our department after he had discovered an indolent mass throughout his epigastric region. He had a history of two melanomas: one invasive melanoma on his left shoulder (Breslow index, $1.4 \mathrm{~mm}$ ) and one melanoma in situ on his back. Total-body CT scans were performed that revealed one metastasis with a size of $1.2 \times$ $2.2 \mathrm{~cm}$ in the lung and one metastasis with a diameter up to $16 \mathrm{~cm}$ in the left upper abdominal cavity. A CT scan of the brain revealed a single hyperdense metastasis with a diameter of $0.9 \mathrm{~cm}$ in the left cerebral hemisphere, which was confirmed using an MRI scan. A punch biopsy of the abdominal lesion was taken confirming metastatic melanoma. Mutational analysis of tissue from the primary invasive melanoma as well as from the abdominal punch biopsy revealed the presence of a BRAFK601E mutation. Due to the high tumor load and rapid progression of the disease, systemic treatment was recommended.

Because BRAF and MEK inhibitors have only been approved for use in cases of BRAFV600-mutated melanomas, and the clinical effect of BRAF inhibition in a patient with this particular mutational status was unclear, we decided to initiate treatment with the off-label use of the MEK inhibitor trametinib (Mekinist ${ }^{\mathrm{TM}}$, Novartis Austria, Vienna, Austria).

Fourteen days after initiating the treatment, the patient felt significantly better and showed further improvement over the following weeks. After 2 months, a control CT scan indicated the partial regression of the abdominal metastasis, and no change in the diameter of the brain and lung metastases. Unfortunately, shortly afterwards the patient displayed radiological progression and experienced intra-abdominal pain. We, therefore, altered the therapy and prescribed nivolumab, but the therapy was terminated by the patient himself due to the increasing pain.

Case 2

A 67-year-old male patient presented himself to his general physician with a bleeding nodule on his right shoulder blade. A shave biopsy was performed and a histopathological 


\section{Case Reports in Oncology}

investigation revealed the presence of an ulcerated melanoma (Breslow index, $1.0 \mathrm{~mm}$ ). Total excision of the remaining tumor as well as a sentinel lymph node biopsy of the right axillary lymph nodes was performed, which revealed the remnants of the melanoma and two negative sentinel nodes. One month later, he developed a highly suspicious lymph node in the left axilla that was confirmed through a histological examination to be a melanoma metastasis. The left axillary region was subsequently excised. The patient received an adjuvant immunotherapy with a low dose of interferon $\alpha_{2 a}$ (Roferon ${ }^{\circledR}-A$, Roche, Austria) for 10 months, but additional lymph node metastases appeared in the left axilla. Two months after the resection, lung metastases were discovered and surgically removed, but relapsed after a few months. The patient entered a phase II, individualized, sensitivity-directed chemotherapy trial which included the use of different chemotherapeutic agents and was randomized to the dacarbazine arm. After three cycles, the tumor load in the patient progressed. The mutational status of the primary melanoma was investigated, and a rare BRAFG466E mutation was detected using Ion Torrent Ampliseq. As little is known about this BRAF mutation, we decided to treat the patient with ipilimumab (Yervoy ${ }^{\mathrm{TM}}$, Bristol-Myers Squibb, Vienna, Austria), since nivolumab and pembrolizumab had not yet been approved in Austria. The patient's disease unfortunately progressed and the performance status of the patient declined rapidly. He was admitted to a supportive care environment and died soon afterwards.

\section{Discussion}

Greater availability of NGS is likely to result in the detection of more rare, 'nonstandard' mutant BRAF melanomas, which raises several issues with regard to treatment and workforce placement [1].

In our department, three questions arose when the rare mutational status was detected in 2 of our patients: first, should we use a BRAF inhibitor that has not been clinically tested for use with such mutations; second, should we use a MEK inhibitor instead, and third, should we try a combination of both?

It is important to note that not every missense mutation in the BRAF gene leads to the increased activation of the BRAF kinase. Several mutations, including the G466E mutation, actually lead to a decrease in activity [6, 7]. In the case of such mutations, the use of a BRAF inhibitor would have been inappropriate, but if it had been used, it may not have benefited the patients, may have altered the pathway activity in a negative way, or delayed the initiation of other treatment options [8].

With respect to case 1, in vitro experiments by Dahlman et al. [9] showed that mutant K601 and L597 293H cells are sensitive to BRAF (vemurafenib) and MEK inhibitors (trametinib), respectively. The use of the MEK inhibitor alone resulted in a more dramatic decrease in the phosphor-ERK protein levels as compared to the use of the BRAF inhibitor alone.

In clinical studies, the positive responses of patients with these rare mutations have been demonstrated by two research groups who favored the use of the MEK inhibitor trametinib, as we did in case $1[10,11]$.

With respect to case 2, Garnett et al. [12] showed that the BRAFG466E and other mutations can activate the MEK-ERK pathway by activating the BRAF homologue CRAF. Smalley et al. [13] reported that melanoma cells harbor a form of the mutated BRAF kinase that has a low activity and are resistant to MEK inhibitors. Furthermore, they showed that apoptosis could be induced by a CRAF inhibitor in these cells. 
The possibility to treat such patients with a CRAF inhibitor needs to be investigated in further clinical trials, and the blockade of various immune checkpoints may offer new potentials for treatment in such patients. Therefore, we decided to treat the second patient with ipilimumab.

\section{Conclusions}

The increasingly frequent use of NGS will play an important role in the identification of rare and 'nonstandard' BRAF mutations, which will raise the question regarding possible targeted treatments. Patients with rare BRAF mutations that result in increased MAP kinase pathway activity may benefit from treatment with MEK inhibitors, whereas patients with rare BRAF mutations that display low MAP kinase activities may benefit from treatment with inhibitors of various immune checkpoints.

\section{Statement of Ethics}

The authors have no ethical conflicts to disclose.

\section{Disclosure Statement}

The authors declare that they have no competing interests and no conflicts of interest.

\section{References}

1 Wilson MA, Morrissette JJ, McGettigan S, et al: What you are missing could matter: a rare, complex BRAF mutation affecting codons 599, 600, and 601 uncovered by next generation sequencing. Cancer Genet 2014;207:272-275.

2 Long GV, Menzies AM, Nagrial AM, et al: Prognostic and clinicopathologic associations of oncogenic BRAF in metastatic melanoma. J Clin Oncol 2011;29:1239-1246.

-3 Chapman PB, Hauschild A, Robert C, et al: Improved survival with vemurafenib in melanoma with BRAF V600E mutation. N Engl J Med 2011;364:2507-2516.

-4 Eisen T, Ahmad T, Flaherty KT, et al: Sorafenib in advanced melanoma: a phase II randomised discontinuation trial analysis. Br J Cancer 2006;95:581-586.

5 Ascierto PA, Minor D, Ribas A, et al: Phase II trial (BREAK-2) of the BRAF inhibitor dabrafenib (GSK2118436) in patients with metastatic melanoma. J Clin Oncol 2013;31:3205-3211.

6 Wan PT, Garnett MJ, Roe SM, et al: Mechanism of activation of the RAF-ERK signaling pathway by oncogenic mutations of B-RAF. Cell 2004;116:855-867.

7 Kamata T, Hussain J, Giblett S, et al: BRAF inactivation drives aneuploidy by deregulating CRAF. Cancer Res 2010;70:8475-8486.

8 Hatzivassiliou G, Song K, Yen I, et al: RAF inhibitors prime wild-type RAF to activate the MAPK pathway and enhance growth. Nature 2010;464:431-435.

$>9$ Dahlman KB, Xia J, Hutchinson K, et al: BRAF(L597) mutations in melanoma are associated with sensitivity to MEK inhibitors. Cancer Discov 2012;2:791-797.

-10 Kim KB, Kefford R, Pavlick AC, et al: Phase II study of the MEK1/MEK2 inhibitor Trametinib in patients with metastatic BRAF-mutant cutaneous melanoma previously treated with or without a BRAF inhibitor. J Clin Oncol 2013;31:482-489.

11 Bowyer SE, Rao AD, Lyle M, et al: Activity of trametinib in K601E and L597Q BRAF mutation-positive metastatic melanoma. Melanoma Res 2014;24:504-508.

12 Garnett MJ, Rana S, Paterson H, et al: Wild-type and mutant B-RAF activate C-RAF through distinct mechanisms involving heterodimerization. Mol Cell 2005;20:963-969.

13 Smalley KSM, Xiao M, Villanueva J, et al: CRAF inhibition induces apoptosis in melanoma cells with non-V600E BRAF mutations. Oncogene 2009;28:85-94. 